\title{
Developmental Impact Analysis of an ICT-Enabled Scalable Healthcare Model in BRICS Economies
}

\author{
Punit Saurabh, Bhaskar Bhowmick, Amrita, and Dhrubes Biswas
}

\author{
66 \\ Age considers; youth ventures. \\ Gurudev Ravindranath Tagore (1861-1941) \\ Noble Laureate, Poet, and Indian Freedom Fighter
}

\begin{abstract}
This article highlights the need for initiating a healthcare business model in a grassroots, emerging-nation context. This article's backdrop is a history of chronic anomalies afflicting the healthcare sector in India and similarly placed BRICS nations. In these countries, a significant percentage of populations remain deprived of basic healthcare facilities and emergency services. Community (primary care) services are being offered by public and private stakeholders as a panacea to the problem. Yet, there is an urgent need for specialized (tertiary care) services at all levels.
\end{abstract}

As a response to this challenge, an all-inclusive health-exchange system (HES) model, which utilizes information communication technology (ICT) to provide solutions in rural India, has been developed. The uniqueness of the model lies in its innovative hub-andspoke architecture and its emphasis on affordability, accessibility, and availability to the masses. This article describes a developmental impact analysis (DIA) that was used to assess the impact of this model. The article contributes to the knowledge base of readers by making them aware of the healthcare challenges emerging nations are facing and ways to mitigate those challenges using entrepreneurial solutions.

\section{Introduction}

Brazil, Russia, India, China, and South Africa (BRICS; tinyurl.com/3s7uxnn) form a conglomerate of the world's fastest-developing large economies. With the rising economic growth of the BRICS nations, the need for healthcare amenities has also grown by leaps and bounds. The challenge is to address the current healthcare issues of people living at the "bottom of the pyramid" in India and other emerging countries (tinyurl.com/3pdssv).

Health is a primary area of concern in BRICS nations, where services are not at par with developed nations. Governments in these nations have resorted to launching several customized health-centered programs and enhancing the basic healthcare infrastructure to bridge the gaps. Unfortunately, the issues relating to healthcare are too large to be addressed by governments alone. Health-service delivery in emerging nations, especially in a diverse country such as India, is under significant pressure. There is a lack of healthcare infrastructure, notably hospitals, primary healthcare facilities, and trained medical manpower (doctors, nurses, etc); there is also a lack of "affordable, available, and accessible" medical services and medicines. Rapid, uncontrolled urbanization and an overdependence on conventional business processes have made the existing healthcare-delivery model in India non-scalable in non-metro and rural areas (Sharad et al, 2011; tinyurl .com/82gemb5). 


\title{
An ICT-Enabled Scalable Healthcare Model in BRICS Economies
}

\author{
Punit Saurabh, Bhaskar Bhowmick, Amrita, and Dhrubes Biswas
}

In India, most of the existing healthcare infrastructure consists of government-supported facilities, Accredited Social Health Activists (ASHA) and individual Registered Medical Practitioners (RMPs), mostly located in smaller towns and rural areas. Primary healthcare support is delivered through a constellation of over 146,000 public healthcare centers and around 4200 community healthcare centers. The Ministry of Health and Family Welfare (mohfw.nic.in) also supports many mid-level hospitals with variable capacity between 50 to 500 beds, as well as district headquarters. Most of the private-sector health infrastructure is located in larger towns and cities. This has forced the smaller city, towns and villages to rely more on government facilities and hospitals with low to medium levels of support services available.

A potential solution to this healthcare challenge is a delivery system based on a unique health-exchange system (HES) model that is scalable and affordable to its users to a considerable extent (Biswas et al. 2010; tiny url.com/6ou6o4g). The term "health-exchange system" is similar to an Indian employment-exchange system (tiny url.com/6vn43du), which provides a platform for the registration of unemployed youths, useful information, routing government training, and supported programs and employment opportunities. In the case of the HES, the model not only provides a platform for the exchange of useful health information, but it also delivers the requisite diagnostic, medical, emergency services required by patients at a nominal charges. Similar such health-exchange organizations exist elsewhere, such as in the United Kingdom (healthexchange.org.uk).

The idea behind the HES was to create business opportunities, infrastructure facilities, and employment opportunities in healthcare, all while providing the targeted population with the required healthcare support. The HES is in tune with Government of India's vision of fostering a grassroots, bottom-up approach to innovation and development and to arrive at healthcare solutions for local problems that are sustainable and scalable (Pande, 2012; tinyurl.com/ce942pc). The next part of the article provides background on the HES, followed by a description of the developmental impact analysis on the model.

\section{The Health-Exchange System}

The HES is a low-cost, scalable healthcare delivery model designed to serve the semi-town and rural areas of West Bengal, India. The HES model was inaugurated in 2009 and is currently operational at eight locations. It involves an innovative healthcare architecture consisting of health kiosks and is strategically located in semiurban and rural areas (Sharad et al., 2011; tinyurl.com/ 82gemb5). The model is spread across tertiary towns and cities far from the reach of mainstream medical facilities but close to the targeted users, who are generally secluded from healthcare infrastructure. The kiosk model is considered inexpensive; it provides several emergency facilities at low cost. Medicines, vital medical measurement devices (e.g., blood pressure and blood sugar meters, electrocardiography and ambulance services are available for the benefit of patients at the kiosk.

The HES is based on an innovative business-process concept which was conceptualized by professors and students of the Indian Institute of Technology Kharagpur (iitkgp.ac.in) under the platform of the Society of Social Entrepreneurs (SSE; sseglobal.com). SSE is a not-for-profit body that brings scalable healthcare services and insurance to people living at "bottom of the pyramid". It established the entrepreneurship-driven HES with intention to provide "global solutions to local problems".

The HES uses a hub-and-spoke architecture, which increases the accessibility of healthcare for the masses without compromising on quality (Amrita et al., 2009; RVIM Journal of Management Research, vol 1:41-56). It consists of a super-specialty hub hospital surrounded by a constellation of "health technology business nodes". These health technology business nodes are commonly referred as "health kiosks" (see Figures 1 and 2). The technology business nodes or kiosks become focal points for creating a business infrastructure

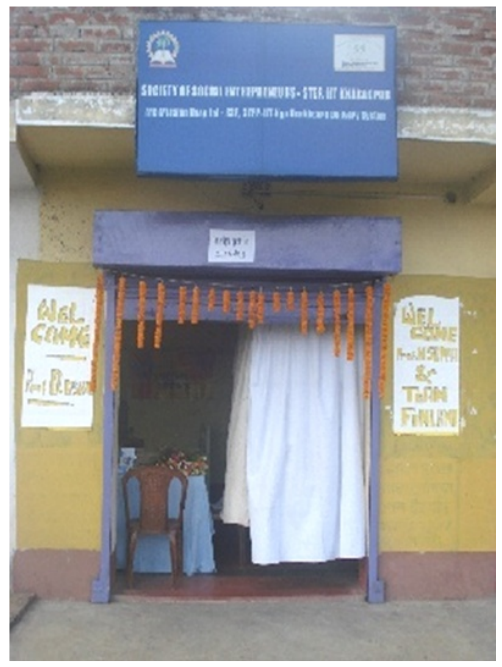

Figure 1. Outside a typical HES kiosk West Bengal, India 


\section{An ICT-Enabled Scalable Healthcare Model in BRICS Economies}

\section{Punit Saurabh, Bhaskar Bhowmick, Amrita, and Dhrubes Biswas}
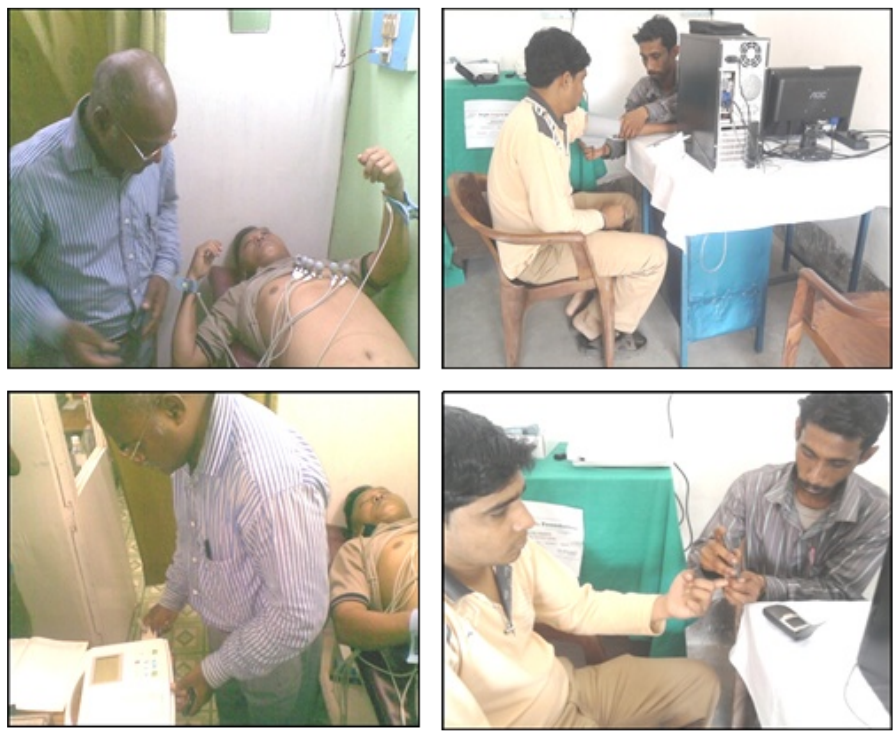

Figure 2. Inside a typical HES kiosk in West Bengal, India

of medical, ambulance, diagnostic, and referral facilities. They also provide emergency healthcare services, maternity services, pre-natal and post-natal services, epidemic response services, etc. At times, these kiosks are also used for immunization, public health awareness, and other related services.

The HES also has alliances with pharmaceutical companies, insurance providers, medical service providers (e.g., hospitals and nursing homes), and educational institutions such as the Indian Institute of Technology Kharagpur for mentoring. These partners help provide accessible and readily available quality healthcare at affordable costs.

The HES model contains three subsystems:

1. Hardware: client tools, servers, mobile access points, etc.

2. Software: database management systems, enterprise resource planning architecture, simulated workflow software and operating systems, optimized health information systems, cloud computing, etc.

3. Manpower: entrepreneurs, researchers, doctors, and academics

The HES model embodies the concept of "equity ownership of entrepreneurs" to deliver public health ser- vices to tertiary towns and cities, and it utilizes private enterprise network platforms supported by the SSE. The HES is designed to establish a unique and vigorous health delivery network at low cost; the kiosks offer innovative and essential health-centric services.

\section{Developmental Impact Analysis}

To scale up the HES model, early feedback for evaluation and path correction was needed in case a flaw in the model was detected. Therefore, a developmental impact analysis was conducted, which can predict the impact of a project before its implementation in the local community. A development impact analysis can also assess the post-development impact of a project on a community. According to Mary Edwards (2000; tinyurl.com/789anot):

"The DIA process makes use of existing information, where possible to determine potential impacts of a proposed development employing techniques to gather additional, new information, where necessary while providing a framework to integrate these data, models, spatial and statistical analyses and experiences in other locales to predict development impacts."

For emerging nations, which have less resources and financial freedom to improve healthcare infrastructure, the development impact analysis assumes greater significance because it helps save precious resources. Thus, the analysis was particularly important for the HES model as it would enable stakeholders to assess the impact of future growth and assist with planning.

To conduct the development impact analysis, the research team visited eight health kiosks set up under the HES model in West Bengal, India. Researchers directly interacted with all the stakeholders of the HES, including the patients, doctors, kiosk owners, managers, and pharmaceutical and equipment suppliers. Particular attention was paid to studying the day-to-day working of the system and the means by which customers were attracted, services were provided, and feedback was received by the kiosk users. Reports generated by government agencies and internal reports relating to kiosks functioning and management were also analyzed.

The development impact analysis considered four key aspects, the results for which are described in the subsections that follow: 


\title{
An ICT-Enabled Scalable Healthcare Model in BRICS Economies
}

\author{
Punit Saurabh, Bhaskar Bhowmick, Amrita, and Dhrubes Biswas
}

1. Technical impact

2. Potential market

3. Fiscal impact

4. Socio-economic impact

\section{Technical impact}

Innovative healthcare products for the HES form an integral part of the business design architecture of the kiosks. A technical analysis of the instruments, devices, and components contributes to the overall aim of developing business architecture and intelligence for the HES.

Cloud-computing technology is used extensively to share resources securely within the network and to provide healthcare providers with flexible access to resources. The kiosks are also equipped with wireless communication devices and an IT infrastructure for basic healthcare facilities, emergency medicines, and testing services. The system installed at the kiosk monitors and delivers a patient's physiological readings to the hospitals and provides an alert mechanism triggered by the patient's vital signs. This information is linked to a medical practitioner's mobile device for analysis. Thus, a doctor can remotely access all the information needed to monitor a patient.

The kiosks also include multifunctional medical instruments that are integrated with Android-based devices (see Figures 3 and 4). Thus, medical data can be collec- ted and relayed by the devices to a centralized database via the GSM mobile communications network (Sharad et al., 2011; tinyurl.com/82gemb5). Enterprise resource planning software (tinyurl.com/8yr9k) combines data from these devices with data provided by healthcare providers. The types of data these instruments can collect include electrocardiographs, blood pressure, pulse rate, plethysmographs, pulse-oximeters, and phono-cardiograms - all integrated with a single mobile device (Tiyashi et al., 2009; tinyurl.com/70stm4s). The device is portable, meaning it can be carried from the kiosk to a patient's house if needed. Although these devices are expensive, the implementation becomes cost effective through volume purchasing.

\section{Potential market}

Looking at the robustness of the model, we believe that it provides tremendous scope and value for money for several stakeholders. The HES model is not only applicable for developing nations in the BRICS region but for nations in Sub-Saharan Africa, Latin America, and the Asia-Pacific region as well. According to the Global Entrepreneurship Monitor (2010; tinyurl.com/77jakrk), there is a considerable percentage of Africans who intend to start their own enterprises and healthcare is one of the priority markets for them.

The socio-economic impact of the model and the resultant effect on participating stakeholders is also important. The model has an elaborate list of partners, including: the kiosk owner or the entrepreneur, registered medical practitioners (RMPs), doctors, hospitals, pharmaceuticals, and manufacturers of mobile

\section{Hardware}

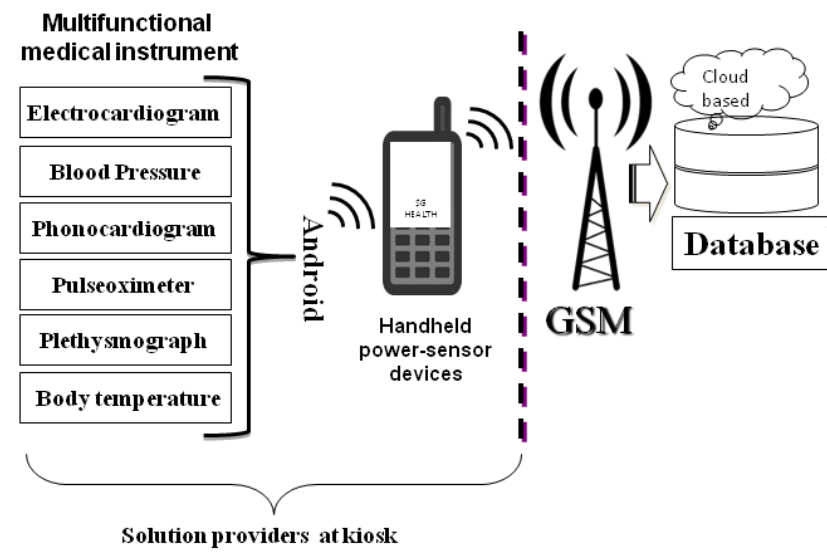

Figure 3. The hardware and software interface devices and architecture in the HES model

\section{Software}
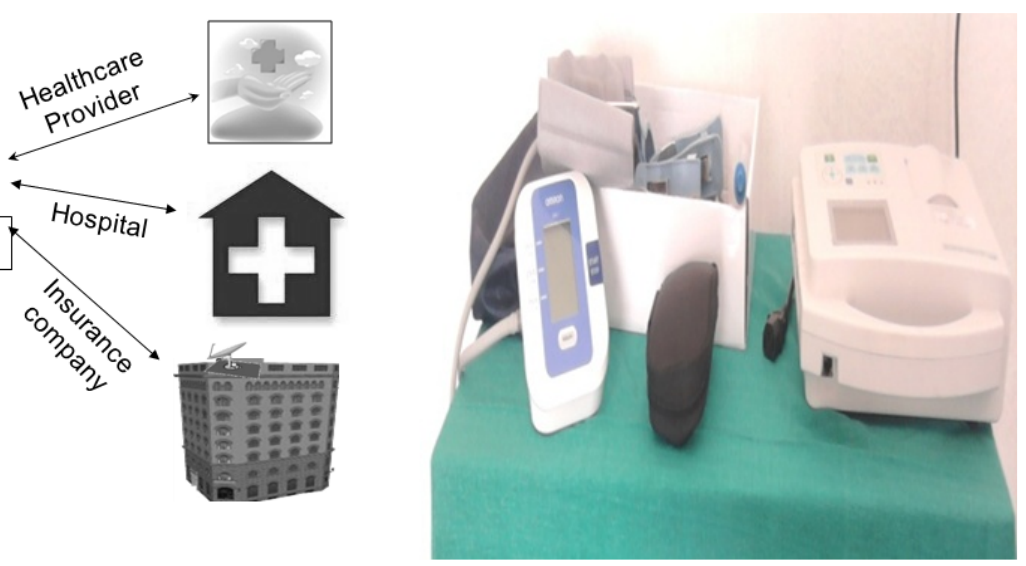

Figure 4. Examples of medical instruments available in an HES kiosk 


\title{
An ICT-Enabled Scalable Healthcare Model in BRICS Economies
}

\author{
Punit Saurabh, Bhaskar Bhowmick, Amrita, and Dhrubes Biswas
}

health devices. Innovators, academics, business providers, researchers, and health professionals can also benefit in their own way from the implementation of the HES model. The HES acts as a platform for associated stakeholders to sell their products and utilize the business opportunities available at the kiosk for their growth. The potential for growth is also high for those companies involved in medical and educational content development, making the HES model a marketfriendly business proposition.

\section{Fiscal impact}

According to Edwards (2000; tinyurl.com/789anot):

"Fiscal analysis involves assessing the public service costs and revenues associated with the development. According to the researcher, such an analysis projects the net cost of the development on the fiscal balance sheet of the community. Since finance plays an important role in determining whether or not to proceed with a proposed development, fiscal impact analysis is a critical component of any development impact assessment".

A properly conceptualized financial model helps generate larger profits, which allow improvement in health infrastructure. This directly impacts the social aspects of the model, thereby strengthening its foundation. Social profits increase inclusivity, which ultimately decreases the incremental operating expenses that are necessary for affordable price points. Further innovations help create a synthesis between branding, insurance price models, and scale-up strategies with a host of partners from healthcare industries as well as from hardware and software organizations. The fiscal impact analysis of the model helps us to understand the fiscal management side of the model implementation and its impact on the market and stakeholders. It is difficult to predict the ultimate financial model for a project before it has been implemented. A properly conceptualized and planned model helps reduce the risk to a considerable extent.

\section{Socio-economic impact}

A socio-economic impact analysis, according to Edwards (2000; tinyurl.com/789anot), measures the potential socio-economic impacts of a development and the changes occurring due to several factors. These factors include demographics, market analyses, public services, and employment and income levels, along with the aesthetic quality of the community. Thus, in order to provide and deliver appropriate medical care, effective planning and management of health services is essential.
The team involved in scaling up the HES is powered by members of the local population who are trained to become entrepreneurs with support from several agencies. The training was provided by the Indian Institute of Technology Kharagpur through the government-supported Technology Entrepreneurship Development Program (TEDP; tinyurl.com/7aozuc9), with handholding from the SSE. The emphasis is on a globally collaborated solution (i.e., following the doctrines of knowledge capital, co-creation and, co-entrepreneurship) by liaising with its partners in Finland (JYU) and the United States.(UCB) Apart from a cost-effective and scalable architecture for tertiary and secondary healthcare delivery, HES is pioneering the creation of the "highways to health" that are essential for all-inclusive economic development of emerging economies, which public funding has so far failed to create. The novelty of TEDP entrepreneurs and optimized global solutions through local involvement enables the HES to more efficiently deliver health services. The HES model has further invoked time-tested methods of microfinance and secondary business solutions to increase affordability for the participants. It has also led to a significant increase in network penetration.

The HES model has benefited its target customers and promoters to a considerable extent. The model provides a low-cost, private alternative that is delivered by local entrepreneurs. The model is co-created by global partners, with significant opportunities for several key players in the proposed "highways to health", spawning hundreds of micro-enterprises that will create rapid economic growth. The ensuing economic growth will ensure that major healthcare gaps in the local community are filled. So far, eight financially successful micro-enterprises have been created and several others are due to become operational shortly. Once the HES model is fully implemented in another five years from now, it will be able to create and sustain more than 5000 local jobs (direct and indirect).

\section{Implications for BRICS Countries and Beyond}

There is considerable scope to replicate the HES model in other BRICS nations and other countries. For example, the model has prospects in Africa and Latin America, which are vast and include many remote areas that are secluded from health care infrastructure. Russia has inaccessible places that hold a considerable population of elderly people that need substantial healthcare support. Similar such conditions exist in several parts of Scandinavia, central Asia, Europe, and America (notably Arizona, Colorado, and New Mexico). 


\title{
An ICT-Enabled Scalable Healthcare Model in BRICS Economies
}

\author{
Punit Saurabh, Bhaskar Bhowmick, Amrita, and Dhrubes Biswas
}

In such areas, there is a need to provide quick and effective healthcare services. These places can effectively be served through the scalable HES model. The model, while creating direct benefits in the local region, indirectly helps the community by creating business opportunities across all stakeholders.

\section{Conclusion}

The uniqueness of the HES model lies in its emphasis on affordability, accessibility, and availability, which has been realized through ICT-driven healthcare technology. Although healthcare is a priority sector for the Indian government, the major factor that motivates the mission to improve healthcare is the affordability of ICT. India has made remarkable progress in this regard and now holds the top rank in terms of the affordability of ICT in the "Global Information Technology Report" (INSEAD-WEF, 2012; tinyurl.com/88fpfjx). This progress has implications for the entire health sector because government and industry usage and emphasis on the affordability of ICT will benefit the health sector (Saurabh, 2012; tinyurl.com/7x4t905).

A review of existing literature shows that a development impact analysis has not been performed on a live, healthcare-based project, which adds a unique perspective to this article. While making an honest assessment of the model, the development impact analysis identified several issues that have a positive or negative impact on the stakeholders in terms of one or more related areas. This analysis thus helps us identify common issues that arise when implementing a project and solving its problems using a case-by-case approach. The article has also highlighted gaps in the literature, which help us understand how we can advance the theory and practice of the HES model in the context of BRICS and other nations.

On the academic side, the HES model has wider ramifications for prospective social entrepreneurs. Initially, the HES model was conceptualized as a joint research initiative of professors and researchers at the Indian Institute of Technology Kharagpur with limited academic output expected. Later, it became a multi-university collaborative effort under the Global Venture Lab platform (gvl3.com) with the University of Jyväskylä in Finland (jyu.fi/en/) and the University of California, Berkeley in the United States (berkeley.edu) as partners. The very fact that academic research initiatives can be tuned in with innovation to create a social enterprise model at a global scale is a success in itself.

\section{About the Authors}

Punit Saurabh is a senior researcher from the Vinod Gupta School of Management at the Indian Institute of Technology Kharagpur. His research specialization includes entrepreneurship and innovation technology management. He is also a research partner at Global Venture Lab (GVL). He has played an instrumental role in the successful establishment and functioning of the DSIR-run TePP Outreach Center at IIT-Kharagpur, providing innovation funding support to individual innovators. At the Center, he has overseen the development and commercialization of more than 30 path-breaking innovations and the functioning of several other innovation and entrepreneurship support programs. As a mentor to startup companies, he provides expert advice and active support to several university-based startups.

Bhaskar Bhowmick is a faculty member at the Rajendra Mishra School of Engineering Entrepreneurship at the Indian Institute of Technology Kharagpur. He is mentoring the dual-degree students in building their career as entrepreneurs. He is also guiding research scholars engaged in studies of business intelligence, business architecture, product development, and social media. His domain of focus is designing an ICT-driven innovation platform in an emerging-country context. He has written papers, cases, book chapters with peers in academia, and presented papers in international conferences. $\mathrm{He}$ is presently focusing on building a model of Education-Entrepreneurship-Enterprise-Environment relating to issues specific to emerging countries.

Amrita is a Research Scholar in the Rajendra Mishra School of Engineering Entrepreneurship at the Indian Institute of Technology Kharagpur. She also oversees the incubation and entrepreneurship support program functioning under SRIC as a Senior Project Officer. She is actively engaged in the study of business intelligence in healthcare for future generations. Her other important areas of research are social media in healthcare. The setting of her research is focused on emerging nations such as India. She has played an active part in the health project implementation by the Society of Social Entrepreneurs (SSE), acting as an enabler of transformation for societal juncture for solving local problems by local solutions. 


\title{
An ICT-Enabled Scalable Healthcare Model in BRICS Economies
}

Punit Saurabh, Bhaskar Bhowmick, Amrita, and Dhrubes Biswas

\begin{abstract}
About the Authors (continued)
Dhrubes Biswas is a Professor of Electronics \& Electrical Communication Engineering, Head of the Rajendra Mishra School of Engineering Entrepreneurship, Professor-in-Charge of Incubation and Entrepreneurship, and Managing Director of Science and Technology at the Entrepreneurs' Park at the Indian Institute of Technology Kharagpur. He directs international university collaborations, technology parks, cross-functional business incubation, the Technology Business Incubator for Innovation and Entrepreneurship (Govt. of India). He also coordinates the Technopreneur Promotion Program for Innovation grants (Govt. of India) and the Technology Entrepreneurship Development Program for grassroots entrepreneurs (Govt. of India). He has championed advanced research in "beyond Moore's" electronic and optical devices in Metamorphic HEMT/ HBT, \& SiGe devices at his nationally acclaimed "India Innovation Semiconductor Fab" at IIT in compound semiconductors. He is an internationally recognized expert in radio frequency integrated circuits (RFIC) and in technology ventures in wireless electronics, cellular phone systems, and communication-related RFICs.
\end{abstract}

ICT-Enabled Scalable Healthcare Model in BRICS

Economies. Technology Innovation Management

Review. June 2012: 25-31. 